\title{
Has the time come for regionalization of surgery for acute type A dissection?
}

\author{
Ivancarmine Gambardella, MD, Christopher Lau, MD, and Leonard N. Girardi, MD
}

The regionalization of complex processes to maximize the opportunity for a positive result is a concept recognized more than 2 millennia ago. During the fourth century, BC, Athenian philosopher Plato stated: "All things will be produced in superior quantity and quality, and with greater ease, when each man works at a single occupation, in accordance with his natural gifts, and at the right moment, without meddling with anything else." Centuries later, industrial economics recognized the association between the frequent performance of intricate operations and success. Wright ${ }^{1}$ described an experience effect in producing airplanes, noting the logarithmic decline in unit costs as a function of cumulative production experience. Although it seems logical that practice makes perfect when it comes to complex surgical procedures, there was not any real data behind this until 1979. Luft and colleagues ${ }^{2}$ examined in-hospital mortality for coronary bypass grafting (CABG) performed in high-volume (HV) (>200 cases/year) versus low-volume (LV) hospitals. Summarizing the results of more than 27,000 CABG operations performed at 587 hospitals reporting to the Professional Activities Study data system, they found the mortality in LV hospitals to be significantly greater $(10.7 \%)$ than that reported from HV hospitals $(6.1 \%){ }^{2}$ Since then, myriad surgical procedures have been analyzed and the voluminous literature on the topic has even generated systematic reviews of systematic reviews that have found positive volume-outcome relationships for many surgical subspecialties. ${ }^{3}$ Nonetheless, relatively uncommon, complex cardiovascular operations associated with high morbidity and mortality, like those performed for an acute type A dissection (ATAAD), have drawn attention as targets for regionalization (Figure 1). Given that the outcomes vary widely, even in HV aortic centers of excellence, it seems appropriate to examine the data in support of such efforts. The issue is incredibly complex and the conclusions that are drawn are open to interpretation and in need of refinement. Herein, we present an overview

From the Department of Cardiothoracic Surgery, Weill Cornell Medicine, New York, NY.

Received for publication April 6, 2020; revisions received June 29, 2020; accepted for publication June 29, 2020; available ahead of print Aug 5, 2020.

Address for reprints: Leonard N. Girardi, MD, Department of Cardiothoracic Surgery, Weill Cornell Medicine, 525 E 68th St, M-404, New York, NY 10065 (E-mail: lngirard@med.cornell.edu).

J Thorac Cardiovasc Surg 2021;161:1734-7

$0022-5223 / \$ 36.00$

Copyright (c) 2020 by The American Association for Thoracic Surgery

https://doi.org/10.1016/j.jtcvs.2020.06.148

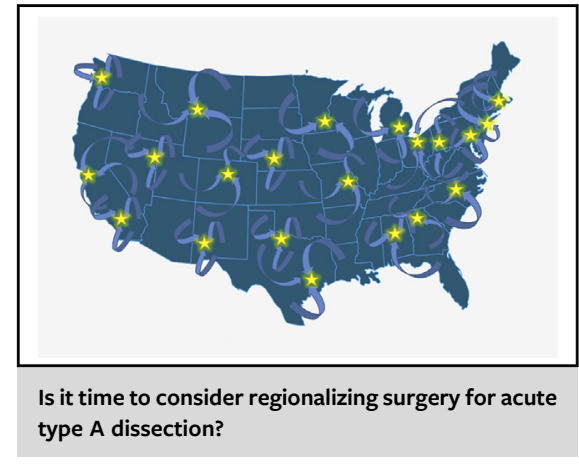

CENTRAL MESSAGE

The benefits of regionalizing surgery for type A dissection depend on patient stability; the geographical density of highvolume centers; and the presence of high-volume, experienced aortic surgeons.

This Invited Expert Opinion provides a perspective on the following paper: Circulation. 2019;140(15) 1239-1250. https://doi.org/10.1161/CIRCULATIONAHA 118.038867.

See Articles on pages 1738 and 1740.

of the contemporary literature regarding regionalization of surgery for ATAAD.

\section{The Nature of the Beast}

ATAAD remains a lethal entity that is almost uniformly fatal without surgical intervention. With an estimated incidence of 3 to 4 per 100,000 population per year, 10,000 to 15,000 US citizens annually will experience this most threatening form of acute aortic syndrome. ${ }^{4,5}$ From the initial onset of the event, mortality is estimated at approximately $1 \%$ to $2 \%$ per hour mostly due to aortic rupture, tamponade, or end organ malperfusion. Half of those experiencing ATAAD will not survive more than 2 days without intervention. ${ }^{6}$ Although modern anti-impulse therapy has become more standardized in managing the acute phase of ATAAD, the mortality associated with nonoperative management remains abysmal. 


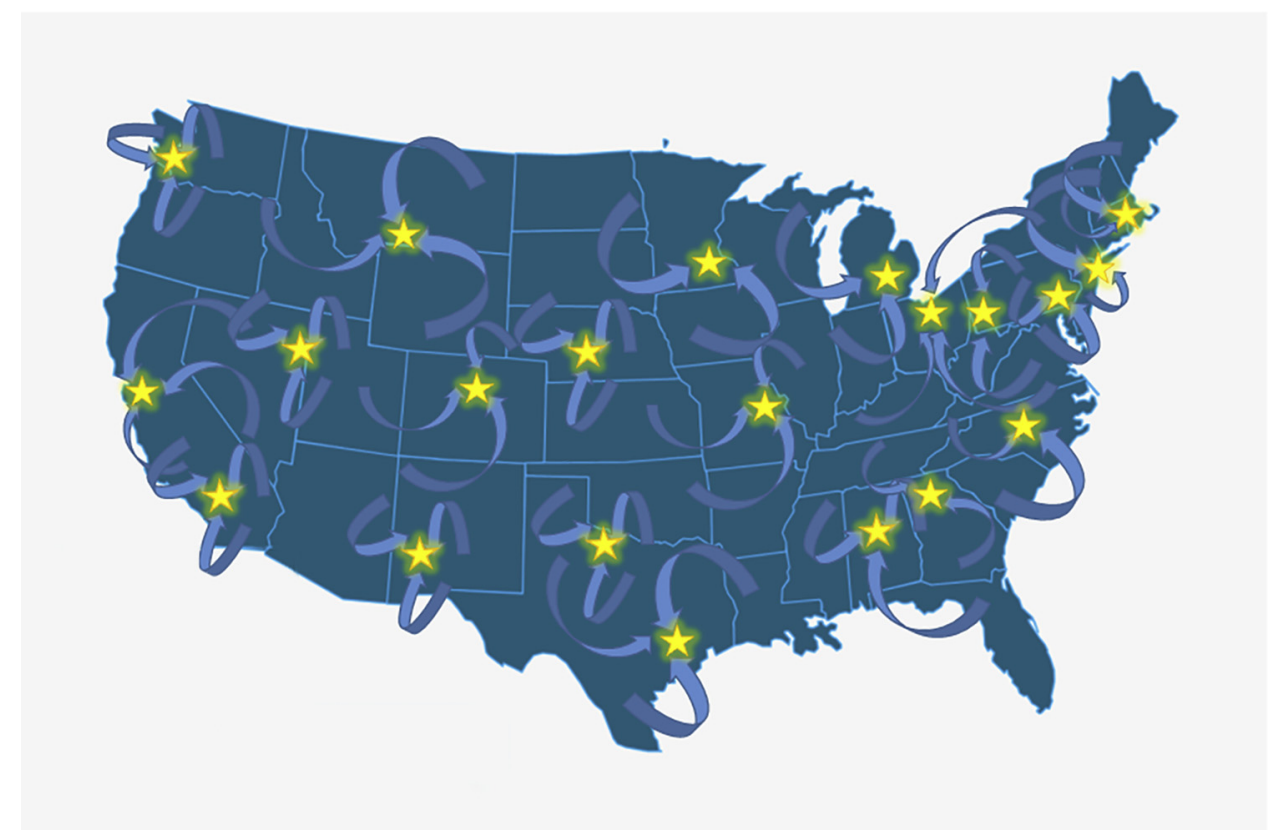

FIGURE 1. Visual map of potential regionalization patterns in the United States.

Conversely, over the past 2 decades across North America and Europe, focused registries like the International Registry of Acute Aortic Dissection (IRAD) show a steady decline in the operative mortality $(\mathrm{OM})$ associated with repair of ATAAD. ${ }^{7}$ Other large datasets like the Nationwide Inpatient Sample of North America (NIS), the National Institute for Cardiovascular Outcomes Research (NICOR) National Adult Cardiac Surgery Audit registry from the United Kingdom and German Registry for Acute Aortic Dissection Type A (GERAADA) confirm that the improvements in outcomes have been seen not only in HV aortic centers of excellence but also in other centers. ${ }^{8-11}$ Despite the positive overall trend, evidence of a volume-outcome relationship persists when HV and LV centers and surgeons are compared.

\section{How Are LV and HV Defined?}

To better comprehend the contribution surgical volume makes to improved outcome for ATAAD, it is crucial to understand the varying definitions of LV and HV for both hospitals and surgeons. In the United States, 2 reports used different methodologies and datasets to arrive at similar conclusions regarding definitions of LV and HV hospitals. Chikwe and colleagues ${ }^{9}$ utilized a 6-year period of the NIS database to identify 5184 patients with an International Classification of Diseases, Ninth Edition Clinical Modification discharge diagnosis of aortic dissection. Institutional volume was divided by the number of years that the hospital was surveyed and was then categorized into quartiles to determine lowest $(<3)$, low $(>3-<8)$, high $(>8-<13)$, and highest $(>13)$ annual hospital volumes. Eleven states participating in NIS also reported individual surgeon volume and similar calculations were performed to divide surgeons into lowest $(<1)$, low $(>1-<2)$, high $(>2-<5)$, and highest $(>5)$ annual volume surgeons. Goldstone and colleagues ${ }^{12}$ scanned the Medicare Provider Analysis Review file over a 15-year period for patients having the same International Classification of Diseases, Ninth Edition Clinical Modification aortic dissection codes as those used to analyze NIS. However, to exclude those patients who may have had type B dissection surgery (an exclusion not performed in the NIS analysis), they used the Current Procedural Terminology codes for surgery of the aortic root and/or the ascending aorta within 14 days of diagnosis or within the same hospitalization. The top decile of hospital volume for these procedures was defined as HV, whereas all others were defined as LV. Although they were not able to stratify LV and HV surgeons from their dataset, the mean annual volume of procedures performed in an HV hospital was 7, a number comparable to the 8 used to define HV from the NIS work by Chikwe and colleagues. ${ }^{9}$ Unfortunately, the NICOR study from the United Kingdom did not define HV and LV hospitals..$^{10}$ It seems reasonable to define an HV hospital as performing a mean annual volume of ATAAD repairs of 7 or more.

When defining an HV ATAAD surgeon, there is a surprising migration toward a common volume as one moves from single-institution experiences toward a complex analysis of national datasets. A contemporary single-center analysis from an $\mathrm{HV}$ aortic center (mean annual proximal aortic volume, 87) showed a marked improvement in results with a mean of 12 annual ATAAD repairs. ${ }^{13}$ Umana-Pizano and colleagues ${ }^{14}$ analyzed their experience with ATAAD repairs in an HV aortic center over a 17-year period. Working backward from an inflection in OM, they defined an HV surgeon as performing $>10$ ATAAD repairs per year. However, their mean annual center experience was 34 ATAAD repairs per year, a uniquely high level of experience when considering 
the median number of ATAAD operations performed at centers participating in the Society of Thoracic Surgeons (STS) database is $3 .^{15}$ The $2 \mathrm{HV}$ surgeons in the Duke group average 6 ATAAD repairs per year-a case volume $>5$ defined those in the highest quartile of experience of those reporting to the NIS database. ${ }^{9}$ Bashir and colleagues ${ }^{10}$ used logistic regression to define annual surgeon ATAAD volume above which $\mathrm{OM}$ significantly declined. A volume of 4.5 cases per year was associated with a significant reduction in OM (odds ratio, 0.853; 95\% CI, 0.773-0.992; $P=.039$ ). Similar to that seen with hospital volume, it seems reasonable to consider an HV ATAAD surgeon as performing at least 5 repairs per year.

\section{How Much Does HV Matter?}

Across the greater community of cardiovascular surgeons performing surgery for ATAAD, it is gratifying to witness a substantial reduction in mortality over time. The IRAD database validates this observation. For 2552 patients undergoing surgery over a 17-year period (1995-2013), the mortality declined from $25 \%$ to $19.7 \%$. $^{7}$ Similar outcomes were reported from the GERAADA database (OM, 20\%) and a more contemporary report from the STS database (OM, $17.3 \%) .{ }^{15,16}$ However, several large datasets have shown a significant volume-outcome relationship. Chikwe and colleagues ${ }^{9}$ reported results from HV surgeons within the NIS database and found that results were significantly better than that achieved by LV surgeons. The HV surgeon in-hospital mortality of $17 \%$ was a significant reduction from the $27.5 \%$ with LV surgeons and more similar to IRAD, GERAADA, or STS outcomes. ${ }^{9}$ The gap between outcomes from HV and LV surgeons reporting to the NICOR registry in the United Kingdom is also supportive of a volumeoutcome relationship. Bashir and colleagues ${ }^{10}$ performed advanced statistical analysis on 1550 ATAAD procedures performed by 249 surgeons over a 6-year period. The in-hospital mortality for HV surgeons was $12.6 \%$, notably lower than the $19.3 \%$ reported by LV surgeons performing < 4 ATAAD repairs per year. Although ATAAD surgery is not intentionally regionalized in the United Kingdom, the number of centers performing these operations is far less than that in the United States. The mean annual hospital volume in the United Kingdom was 9.6, substantially more than the average of 3 reported in the STS database. By our definition of HV hospitals ( $\geq 7$ ATAAD repairs per year), $>80 \%$ of UK hospitals would be considered HV. With this unintentional semiregionalization of ATAAD surgery, hospital volume had little influence over mortality (odds ratio, 1.005; 95\% CI, 0.956-1.057; $P=.84$ ) but the influence of individual surgeon volume persisted.

When one examines the volume-outcome relationship for HV surgeons operating within HV aortic centers of excellence, it becomes more difficult to deny the substantial benefits touted by those in favor of regionalization. An early report from an $\mathrm{HV}$ aortic center hinted at the potential benefits of aligning surgeon and hospital resources in the pursuit of excellence. Within a center averaging in excess of 40 ATAAD operations per year, and with surgeons performing approximately 10 procedures per year, the $\mathrm{OM}$ was $9.8 \% .{ }^{17}$

A deeper dive into the benefits of instituting a focused thoracic aortic surgery program (TASP) further corroborates the advantages of specialization. Andersen and colleagues ${ }^{13}$ compared the results of ATAAD repair before and after establishment of a TASP composed of dedicated surgeons with standardized intraoperative protocols and a multidisciplinary approach to postoperative care. The results were dramatic and the reasons for improvement multifactorial. With a reduction in the number of participating surgeons from 11 to 2 principal surgeons, the mean annual surgeon volume rose from 2 per year to 6 . There was a 12 -fold reduction in mortality from the pre-TASP era mortality from $33.9 \%$ to $2.8 \%$ after the program was reorganized. Substantial reductions were also observed for the incidence of postoperative hemorrhage requiring re-exploration, delayed sternal closure, and the need for concomitant CABG. Although the average number of yearly ATAAD cases experienced only a modest increase from 9 to 12 over the 12-year study period, the mean annual number of total proximal aortic procedures increased to nearly 90. This accumulation of experience with the nuances of ATAAD and the complex surgical procedures required to successfully treat this disease clearly had a positive influence.

Similar to the supposition put forth by Andersen and colleagues, ${ }^{13}$ Umana-Pizano and colleagues ${ }^{14}$ found that total aortic exposure was critical. They corroborated the findings, espousing the institution of an HV surgeon/HV center system. In an aortic center of excellence averaging 34 ATAAD repairs annually over a nearly 30 -year period, they demonstrated a 4-fold increase in hospital mortality when operations within an HV system were performed by LV surgeons. This included accumulated experience on when ATAAD operations could be delayed until daylight hours to avoid the effects of sleep deprivation and to have access to a hospital's full resources. HV surgeons operated an average of 15 hours after patient admission, whereas LV surgeons typically operated within 4 hours of arrival. No patient died while awaiting surgery by an HV surgeon. Of course, patients with ongoing symptoms, hemodynamic compromise, and malperfusion were taken for immediate surgery. However, the potential benefits and documented safety of waiting to operate under optimal conditions (again-for emphasis-on stable patients!) calls into question the $1 \%$ per hour mortality concerns and segues perfectly into the argument in favor of transfer to $\mathrm{HV}$ institutions and surgeons.

\section{What are the Risks and Benefits of Transfer to an HV Center?}

ATAAD is an acute event with presenting symptoms that are similar to acute coronary syndromes, prompting 
patients to present to their local emergency departments, which may not have cardiac surgical services. Thus, more than $60 \%$ of patients treated for ATAAD at experienced centers are transferred from other hospitals. ${ }^{7}$ In the United States, a quarter of all Medicare beneficiaries present to a hospital without open heart surgery (OHS) capabilities, whereas another $50 \%$ present to an LV hospital performing $<7$ ATAAD procedures per year. ${ }^{12}$ For those arriving at a hospital without OHS, transfer is a necessity. However, $40 \%$ are sent to an LV hospital with limited experience in managing ATAAD. An additional $24 \%$ of patients transferred out of LV hospitals are inexplicably sent to another LV hospital. For those undergoing surgery in a, LV hospital, the mortality was $29.9 \%$, significantly higher than the $21.9 \%$ experienced in an HV institution. Clearly, the limitations of the Medicare dataset create inherent bias in this analysis. The stability of patients having surgery at or transferring to LV hospitals is impossible to discern. However, the authors performed a complex sensitivity analysis that demonstrated $4.4 \%$ of patients rerouted to HV hospitals would have to die to negate the advantages of avoiding transfer to an LV hospital. The mean distance between LV and HV hospitals was a mere 50 miles. Fixed-wing transport obviously mitigates this additional distance. Ground transportation, even in populated urban environments, would likely cover these extra miles in 2 hours or less. Given the lack of adverse events associated with delaying surgery in selected patients reported by both Andersen and colleagues ${ }^{13}$ and Umana-Pizano and colleagues,${ }^{14}$ it seems that the extra hour or 2 needed for transfer would have little negative influence on the ultimate outcome. However, transfer to an HV center can benefit patients, with a $7 \%$ to $10 \%$ reduction in mortality as seen in this Medicare study, ${ }^{12}$ the NIS study, ${ }^{9}$ and the NICOR study, ${ }^{10}$ offsetting any risk of the transfer itself.

\section{CONCLUSIONS}

Despite tremendous improvements in operative techniques and perioperative care, surgery for ATAAD is still associated with substantial morbidity and mortality. There is significant opportunity for a better outcome when patients undergo surgery in $\mathrm{HV}$ institutions by surgeons with extensive experience in proximal aortic surgery. Given that the majority of patients experiencing an ATAAD will present to either a center without OHS capabilities or to 1 with limited experience in performing ATAAD procedures, transfer to an HV hospital should be considered, particularly if an HV surgeon is available to perform surgery. An accumulating body of evidence also supports the concept that the limited risks associated with the transfer of stable patients are mitigated by the significant reduction in operative mortality realized in experienced hands.

\section{Conflict of Interest Statement}

The authors reported no conflicts of interest.

The Journal policy requires editors and reviewers to disclose conflicts of interest and to decline handling or reviewing manuscripts for which they may have a conflict of interest. The editors and reviewers of this article have no conflicts of interest.

\section{References}

1. Wright TP. Factors affecting the cost of airplanes. J Aeronaut Sci. 1936;3: 122.

2. Luft HS, Bunker JP, Enthoven AC. Should operations be regionalized? The empirical relation between surgical volume and mortality. N Eng J Med. 1979; 301:1364-9.

3. Morche J, Mathes T, Pieper D. Relationship between surgeon volume and outcomes: a systematic review of systematic reviews. Syst Rev. 2016;5 204.

4. Olsson C, Thelin S, Stable E, Ekbom A, Granath F. Thoracic aortic aneurysm and dissection: increasing prevalence and improved outcomes reported in a nationwide population-based study of more than 14,000 cases from 1987 to 2002. Circulation. 2006;114:2611-8.

5. Clouse WD, Hallett JW Jr, Schaff HV, Spittell PC, Rowland CM, Ilstrup DM, et al. Acute aortic dissection: population-based incidence compared with degenerative aortic aneurysm rupture. Mayo Clin Proc. 2004;79:176-80.

6. Hagan PG, Nienaber CA, Isselbacher EM, Bruckman D, Karavite DJ, Russman PL, et al. The international registry of acute aortic dissection (IRAD): new insights into an old disease. JAMA. 2000;283:897-903.

7. Pape LA, Awais M, Woznicki EM, Suzuki T, Trimarchi S, Evangelista A, et al. Presentation, diagnosis, and outcomes of acute aortic dissection: 17-year trends from the international registry of acute aortic dissection. J Am Coll Cardiol. 2015;66:350-8.

8. Knipp BS, Depp GM, Prager RL, Williams CY, Upchurch GR, Patel HJ. A contemporary analysis of outcomes for operative repair of type A aortic dissection in the United States. Surgery. 2007;142:524-8.

9. Chikwe J, Cavallaro P, Itagaki S, Seigerman M, DiLuzzo G, Adams DH. National outcomes in acute aortic dissection: influence of surgeon and institutional volume on operative mortality. Ann Thorac Surg. 2013;95:1563-9.

10. Bashir M, Harky A, Fok M, Shaw M, Hickey GL, Grant SW, et al. Acute type A aortic dissection in the United Kingdom: surgeon volume-outcome relation. J Thorac Cardiovasc Surg. 2017;154:398-406.

11. Kruger T, Conzelmann LO, Bonser RS, Borger MA, Czerny M, Wildhirt S, et al Acute aortic dissection type A. Br J Surg. 2012;99:1331-44.

12. Goldstone AB, Chiu P, Baiocchi M, Lingala B, Lee J, Rigdon J, et al. Interfacility transfer of Medicare beneficiaries with acute type A aortic dissection and regionalization of care in the United States. Circulation. 2019;140:1239-50.

13. Andersen ND, Ganapathi AM, Hanna JM, Williams JB, Gaca JG, Hughes GC Outcomes of acute type A dissection repair before and after implementation of a multidisciplinary thoracic aortic surgery program. J Am Coll Cardiol. 2014; 63:1796-803.

14. Umana-Pizano JB, Nissen AP, Sandhu HK, Miller CC, Loghin A, Safi HJ, et al Acute type A dissection repair by high-volume vs low-volume surgeons at a highvolume aortic center. Ann Thorac Surg. 2019;108:1330-6.

15. Lee TC, Kon Z, Cheema FH, Grau-Sepulveda MV, Englum B, Kim S, et al. Contemporary management and outcomes of acute type A aortic dissection: an analysis of the STS adult cardiac surgery database. J Card Surg. 2018;33: $7-18$.

16. Easo J, Weigang E, Hölzl PP, Horst M, Hoffmann I, Blettner M, et al. Influence of operative strategy for the aortic arch in DeBakey type I aortic dissection: analysis of the German registry for acute aortic dissection type A. J Thorac Cardiovasc Surg. 2012;144:617-23.

17. Bavaria JE, Brinster DR, Gorman RC, Woo YJ, Gleason T, Pochettino A. Advances in the treatment of acute type A dissection: an integrated approach. Ann Thorac Surg. 2002;74(Suppl):S1848-63.

Key Words: regionalization, aortic surgery, type A dissection, aortic dissection 